\title{
Caracterização epidemiológica da leptospirose suína em criações não tecnificadas do semiárido brasileiro ${ }^{1}$
}

\author{
Alexandro I. Leite ${ }^{2 *}$, Wesley A.C. Coelho ${ }^{2}$, Roberta L.L. Brito ${ }^{3}$, Glaucenyra C.P. Silva ${ }^{4}$, \\ Renata F. Santos ${ }^{4}$, Luis A. Mathias ${ }^{4}$ e Iveraldo S. Dutra ${ }^{5}$
}

\begin{abstract}
Leite A.I., Coelho W.A.C., Brito R.L.L., Silva G.C.P., Santos R.F., Mathias L.A. \& Dutra I.S. 2018. [Epidemiological characterization of leptospirosis in pig herds reared under poor technical condition in the Brazilian semiarid.] Caracterização epidemiológica da leptospirose suína em criações não tecnificadas do semiárido brasileiro. Pesquisa Veterinária Brasileira 38(4):613-619. Departamento de Ciências Animais, Universidade Federal Rural do Semi-Árido, Av. Francisco Mota 572, Costa e Silva, Mossoró, RN 59625-900, Brazil. E-mail: alex@ufersa.edu.br.

This study was conducted in pigs of Mossoró, Rio Grande do Norte, with the objective of epidemiological characterization of swine leptospirosis in herds reared under poor technical and sanitary conditions in the Brazilian semiarid. Serological diagnosis of infection was carried out by means of microscopic agglutination test (MAT), and the samples were submitted to a collection of 24 Leptospira serovars. Information about, variables referring to the farm owner, general property characteristics, productive, reproductive and health management were obtained, by means of visits to the farms and interviews, in order to perform an analysis of risk factors. A prevalence of 78.6\% (324/412) of seropositive pigs was observed. The samples were positive to 15 serovars, with predominance of serovar Icterohaemorrhagiae, followed by Pomona, Shermani and Tarassovi. The animals were positive for up to eight serovars. The titles ranged from 100 to 12,800, and the highest titers were observed to serovar Pomona. In all the 20 examined herds there were reactor animals, and the prevalence in each herd ranged from $25 \%$ to $100 \%$. The risk factors that were associated with leptospirosis were the confinement system of animals and bad sanitation of breeding facilities. The use of treated water for watering the animals showed a protective factor. The results showed that Leptospira spp. was found to be quite widespread in swine farms of Mossoró, with wide variation of circulating serovars, representing a problem in animal production and risks to public health.
\end{abstract}

INDEX TERMS: Leptospirosis, swine health, epidemiology, prevalence, risk factors, Leptospira spp., Brazil, bacterioses.

RESUMO.- Este estudo foi realizado em suínos do município de Mossoró, Rio Grande do Norte, com o objetivo de caracterizar epidemiologicamente a leptospirose suína em criações

\footnotetext{
${ }^{1}$ Recebido em 18 de abril de 2017.

Aceito para publicação em 27 de abril de 2017.

2 Departamento de Ciências Animais, Universidade Federal Rural do Semi-Árido, Av. Francisco Mota 572, Costa e Silva, Mossoró, RN 59625-900, Brasil. * Autor para correspondência: alex@ufersa.edu.br

${ }_{3}$ Instituto Superior de Teologia Aplicada, Rua Antônio Rodrigues Magalhães, Sobral, CE 62050-100, Brasil.

${ }^{4}$ Departamento de Medicina Veterinária Preventiva e Reprodução Animal, Faculdade de Ciências Agrárias e Veterinárias (FCAV), Universidade Estadual Paulista (Unesp), Via de Acesso Prof. Paulo Donato Castellane s/n, Zona Rural, Jaboticabal, SP 14884-900, Brasil.

${ }^{5}$ Departamento de Apoio, Produção e Saúde Animal, Faculdade de Medicina Veterinária (FMV), Unesp, Rua Clóvis Pestana 763, Jardim Dona Amélia, Araçatuba, SP 16050-680, Brasil. E-mail: isdutra@fmva.unesp.br
}

não tecnificadas do semiárido brasileiro. Para isso, foi realizado diagnóstico sorológico da infecção pela técnica de soroaglutinação microscópica (SAM), sendo as amostras submetidas a uma coleção de 24 variantes de leptospira, e foram obtidas informações, por meio de visitas às criações e entrevistas com os proprietários, abordando variáveis referentes a criador, características gerais da propriedade, manejo produtivo, reprodutivo e sanitário, com o propósito de realizar análise de fatores de risco. Obteve-se uma prevalência de 78,6\% (324/412) de suínos soropositivos. As amostras foram positivas para 15 sorovares, com predominância do sorovar Icterohaemorrhagiae, seguido do Pomona, Shermani e Tarassovi. Os animais foram positivos a até oito sorovares. Os títulos variaram de 100 a 12.800, sendo as maiores titulações contra o sorovar Pomona. Em todas as 20 propriedades trabalhadas (100\%) existiam animais reagentes, e a prevalência 
da infecção em cada propriedade variou de $25 \%$ a $100 \%$. Os fatores de risco que estavam associados à leptospirose foram o sistema de confinamento dos animais e o saneamento ruim das instalações de criação. 0 uso de água tratada na dessedentação dos animais se mostrou fator de proteção. Os resultados mostraram que a Leptospira spp. encontrava-se bastante disseminada nas criações de suínos de Mossoró, com grande variação de sorovares circulantes, representando um problema na produção animal e riscos para a saúde pública.

TERMOS DE INDEXAÇÃO: Leptospirose, suínos, epidemiologia, soroprevalência, fatores de risco, Leptospira spp., bacterioses.

\section{INTRODUÇÃO}

A leptospirose é considerada a zoonose mais amplamente distribuída no mundo, com significativo impacto social, econômico e sanitário, tendo sido referida como uma doença infecciosa reemergente, tanto no meio rural quanto no urbano (Higgins 2004). A infecção é provocada por uma espiroqueta do gênero Leptospira, reconhecida como um importante problema de saúde pública, e sua ocorrência é favorecida pelas condições ambientais, em climas tropicais e subtropicais.

Várias espécies de animais domésticos e selvagens são suscetíveis a esta zoonose, podendo apresentar infecções subclínicas e tornarem-se reservatórios, eliminando as leptospiras na urina (Acha \& Szyfres 2001). No ser humano, a leptospirose é uma zoonose de grande importância social e econômica por apresentar elevada incidência em determinadas áreas, com alto custo hospitalar e perdas de dias de trabalho, bem como por sua letalidade, que pode chegar a até $40 \%$ dos casos mais graves (Brasil 2009).

No Brasil, os levantamentos sorológicos para o diagnóstico de infecção por leptospiras em suínos revelaram percentuais que variaram de 4,7\%, nos Estados do Maranhão e Piauí (Gonçalves et al. 2011), até 66,1\%, no Rio de Janeiro (Ramos \& Lilenbaum 2002). Os sorovares Icterohaemorrhagiae, Pomona e Canicola têm sido apontados como os mais importantes na epidemiologia da doença (Soto et al. 2007). No Rio Grande do Norte, estudos sobre a soroprevalência da leptospirose em animais são escassos, tendo sido descritos apenas nas espécies bovina, ovina e caprina (Favero et al. 2001, Azevedo et al. 2004, Araújo Neto et al. 2010). No entanto, trabalhos com a espécie suína ainda não foram realizados.

Na suinocultura, a prevenção da leptospirose é largamente dependente de medidas de saneamento da granja e de diagnóstico da doença, que muitas vezes são difíceis de serem implementadas, principalmente em regiões onde a criação não é tecnificada. Uma granja de suínos oferece múltiplas formas para a viabilidade, permanência e transmissão da leptospirose, pelas características favoráveis do ambiente, do manejo e das instalações (Delbem et al. 2004).

Considerando a complexidade do caráter multifatorial da leptospirose, o maior conhecimento de sua epidemiologia reveste-se de significado para seu controle e consequente diminuição de seus impactos. Diante do exposto, objetivou-se com este estudo caracterizar epidemiologicamente a leptospirose suína em criações não tecnificadas do semiárido brasileiro.

\section{MATERIAL E MÉTODOS}

A pesquisa foi desenvolvida no município de Mossoró, localizado na região do semiárido brasileiro, mais especificamente na Mesorregião Oeste do Estado do Rio Grande do Norte. O município possui uma população de 280.314 habitantes, é o segundo mais populoso, o maior em extensão territorial e concentra o maior rebanho suíno do estado (IBGE 2012a).

Foi realizado um estudo epidemiológico transversal para estimar a frequência e analisar fatores de risco associados à leptospirose suína. 0 tamanho da amostra foi calculado pela fórmula utilizada em epidemiologia de acordo com o número de animais existentes no município, que era de 6.046 suínos (IBGE 2012b), considerando uma prevalência desconhecida, estimada em 50\%, com nível de confiança de 95\% e erro estatístico de 5\% (Thrusfield 2004), o que determinou uma amostra mínima total de 384 animais; assim, foram colhidas 412 amostras.

Para a formação da amostra, buscou-se incluir no estudo animais das 20 principais propriedades de criações comerciais do município, as quais, por sua vez, eram todas não tecnificadas. Nesse sentido, a amostragem foi probabilística, representando a população de suínos do município de Mossoró, RN, e a escolha das propriedades trabalhadas foi por conveniência, por ocasião do abate dos suínos. O quantitativo de suínos por rebanho nas 20 criações variou de 15 a 400 animais, e $55 \%$ das propriedades tinham mais de 100 animais.

A título de categorização neste trabalho, considerou como sistema de criação extensivo aquele em que os suínos eram criados em áreas de terra extensas, totalmente soltos, com acesso as pastagens, poucos insumos, equipamentos e mão de obra. 0 sistema de semiconfinamento se aplicou para aquelas criações em que os animais recebiam algum tipo de suplemento alimentar, tendo acesso a pequenos cercados e à pastagem. Por fim, se considerou como sistema de confinamento aquele em que se tinha um grande número de animais presos em cercados, com toda a alimentação fornecida pelos proprietários e sem acesso a pastagem.

A coleta de sangue foi realizada por meio de punção da veia jugular, no período de janeiro a dezembro de 2013, sendo as amostras dessoradas e estocadas no mesmo dia. Imediatamente após a coleta, o sangue foi transferido para tubos de ensaio, que foram identificados e mantidos em temperatura ambiente até a retração do coágulo. As amostras foram, então, transportadas em caixas isotérmicas, e depois centrifugadas por 10 minutos a 3.000rpm para a obtenção do soro. Depois foram aliquotadas e estocadas a $-20^{\circ} \mathrm{C}$ até a realização dos testes sorológicos.

As propriedades foram visitadas para a realização de inspeção das criações e entrevistas com os produtores, por meio de um roteiro contendo questões abertas e fechadas sobre ambiente, manejo, alimentação, controle sanitário e profilático dos animais, além de dados de ordem humana, social e econômica dos proprietários. Em todos os casos (100\%), inexistia vacinação para leptospirose em qualquer fase da vida dos animais. Neste estudo, foram considerados animais jovens aqueles com idade inferior a 6 meses e adultos aqueles com idade $\geq 7$ meses. Com relação ao saneamento da propriedade, foram estabelecidas 3 categorias: saneamento bom (higienização diária, água tratada usada na dessedentação dos animais e boas condições higiênicas no momento da inspeção às instalações); saneamento regular (higienização diária, água tratada usada na dessedentação dos animais e condições higiênicas insatisfatórias no momento da inspeção às instalações) e saneamento ruim (higienização não diária, água não tratada e condições higiênicas insatisfatórias no momento da inspeção às instalações). Foi considerada uma boa higienização quando era adotada a higienização diária.

Para a pesquisa de anticorpos contra Leptospira spp., foi utilizada a técnica de soroaglutinação microscópica (SAM), de acordo com a metodologia descrita pela OIE (2008). As amostras de soro 
sanguíneo foram diluídas em solução salina, sendo a diluição inicial $1 / 25$. Alíquotas de $25 \mu \mathrm{L}$ foram colocadas em placas de poliestireno, com fundo chato, e adicionada igual quantidade de antígeno, de 24 sorovariedades (Icterohaemorrhagiae, Patoc, Shermani, Pomona, Tarassovi, Bratislava, Autumnalis, Copenhageni, Butembo, Castellonis, Bataviae, Sentot, Pyrogenes, Australis, Hebdomadis, Panamá, Cynopteri, Andamana, Grippotyphosa, Canicola, Wolffi, Hardjo, Whitcombi e Javanica) de Leptospira spp., resultando na diluição de 1/50. A mistura soro-antígeno foi levemente homogeneizada e incubada em estufa BOD à temperatura de $28^{\circ} \mathrm{C}$ por 40 a 120 minutos, procedendo-se à leitura em microscopia de campo escuro, diretamente nos poços da placa. Foram consideradas reagentes as amostras em que se observou 50\% de aglutinação, estando metade das leptospiras aglutinadas no campo microscópico no aumento de 100 vezes. As amostras reagentes na diluição inicial foram testadas com diluições seriadas de razão dois, sendo a primeira diluição 1/100, conforme a recomendação da OIE (2008).

Consideraram-se como animais reagentes aqueles com título igual ou superior a 100. O sorovar considerado como provável causador da infecção foi o que apresentou maior título. Na eventualidade de o maior título ser apresentado contra dois ou mais sorovares, a amostra foi enquadrada como coaglutinação, conforme Delbem et al. (2004), Aguiar et al. (2006), Azevedo et al. (2006), Marques et al. (2010), Figueiredo et al. (2013) e Vieira et al. (2013).

Os dados laboratoriais e da entrevista foram digitados em planilha eletrônica e, após checagem, transferidos para o software estatístico SPSS 17.0 (Statistical Package for the Social Sciences), sendo posteriormente codificados para realização das análises. Diversos grupos foram comparados, obtendo-se odds ratio (OR), intervalos de confiança de 95\%, e significância determinada através do teste do qui-quadrado $\left(\chi^{2}\right)$ e exato de Fisher. Este último, por sua vez, foi utilizado sempre que se verificassem valores com frequência esperada inferior a 5 . 0 nível de significância estabelecido foi o valor de $\mathrm{p}<0,05$.

Para o ajuste das variáveis foi utilizado um modelo de regressão logística para estudar, de forma simultânea, os múltiplos efeitos que poderiam estar envolvidos na prevalência da soropositividade para leptospirose. Compuseram esta etapa da análise as variáveis que tiveram relação com a leptospirose com valor de $\mathrm{p}<0,20$. Em função do número de variáveis estudadas, inicialmente foram montados modelos logísticos para cada grupo, de acordo com características relevantes aos animais e proprietários. Foram excluídas do modelo logístico as que apresentaram intervalos de confiança de grande amplitude nas estimativas da odds ratio, bem como as que apresentaram colinearidades. Sendo assim, compuseram a análise para o modelo final variáveis com nível de significância de p<0,10. Estas, por sua vez, foram reagrupadas em um modelo único e novamente avaliadas pelo método completo de remoção sucessiva das variáveis. Nesta etapa foi utilizado o nível de significância de $\mathrm{p}<0,05$. Como medida de qualidade de ajuste de regressão logística foi utilizada o teste de Hosmer e Lemeshow, no qual um $\mathrm{p} \geq 0,05$ indica que o modelo está ajustado.

O projeto de pesquisa foi aprovado pela Comissão de Ética no Uso de Animais (CEUA) da Universidade Federal Rural do Semi-Árido (UFERSA), registrado sob № 02/2013, e também pelo Comitê de Ética em Pesquisa da Universidade Potiguar, por envolver entrevista com os proprietários dos suínos, com parecer de № 290.224/2013 CEP/UNP.

\section{RESULTADOS}

Dos 412 suínos examinados, 324 reagiram a um ou mais dos sorovares empregados, resultando em uma soropositividade de 78,6\%. Dos 24 sorovares testados, houve suínos reatores a 15. Os títulos variaram de 100 a 12.800 , sendo as maiores titulações contra o sorovar Pomona. Houve elevada ocorrência de coaglutinações $(22,3 \%)$ e predominância do sorovar Icterohaemorrhagiae $(20,2 \%)$, seguido dos sorovares Pomona (11,2\%), Shermani (7,8\%), e Tarassovi $(6,3 \%)$. Também foram constatadas reações sorológicas aos sorovares Patoc $(3,4 \%)$, Autumnalis (1,9\%), Bratislava (1,5\%), Castellonis $(0,7 \%)$, Sentot $(0,7 \%)$, Bataviae $(0,7 \%)$, Copenhageni $(0,5 \%)$, Butembo $(0,5 \%)$, Australis $(0,2 \%)$, Panamá $(0,2 \%)$ e Canicola $(0,2 \%)$ (Quadro 1). Dentre as 20 propriedades trabalhadas, todas

Quadro 1. Número de reações para cada título de aglutininas entre os 324 suínos sororreagentes pelo teste de soroaglutinação microscópica, a uma ou mais das 24 sorovariedades de Leptospira spp., entre 412 suínos, abatidos no período de janeiro a dezembro de 2013. Mossoró/RN, 2013

\begin{tabular}{|c|c|c|c|c|c|c|c|c|c|c|}
\hline \multirow{2}{*}{ Sorovar } & \multicolumn{8}{|c|}{ Títulos } & \multirow{2}{*}{ Total } & \multirow{2}{*}{$\%$} \\
\hline & 100 & 200 & 400 & 800 & 1600 & 3200 & 6400 & 12800 & & \\
\hline Icterohaemorrhagiae & 44 & 31 & 8 & - & - & - & - & - & 83 & 20,2 \\
\hline Pomona & 4 & 13 & 7 & 5 & 8 & 4 & 1 & 4 & 46 & 11,2 \\
\hline Shermani & 9 & 8 & 13 & 2 & - & - & - & - & 32 & 7,8 \\
\hline Tarassovi & 4 & 12 & 8 & 1 & 2 & - & - & - & 27 & 6,6 \\
\hline Patoc & 5 & 5 & 4 & - & - & - & - & - & 14 & 3,4 \\
\hline Autumnalis & 3 & 1 & 3 & - & 1 & - & - & - & 8 & 1,9 \\
\hline Bratislava & 1 & 5 & - & - & - & - & - & - & 6 & 1,5 \\
\hline Castellonis & 1 & 2 & - & - & - & - & - & - & 3 & 0,7 \\
\hline Sentot & 1 & 2 & - & - & - & - & - & - & 3 & 0,7 \\
\hline Bataviae & 2 & 1 & - & - & - & - & - & - & 3 & 0,7 \\
\hline Copenhageni & 1 & - & - & - & 1 & - & - & - & 2 & 0,5 \\
\hline Butembo & - & 2 & - & - & - & - & - & - & 2 & 0,5 \\
\hline Australis & - & 1 & - & - & - & - & - & - & 1 & 0,2 \\
\hline Panamá & - & 1 & - & - & - & - & - & - & 1 & 0,2 \\
\hline Canicola & - & - & 1 & - & - & - & - & - & 1 & 0,2 \\
\hline Coaglutinação* & 62 & 24 & 6 & - & - & - & - & - & 92 & 22,3 \\
\hline Total reações positivas & & & & & & & & & 324 & 78,6 \\
\hline Reações negativas & & & & & & & & & 88 & 22,4 \\
\hline Total examinados & & & & & & & & & 412 & 100,0 \\
\hline
\end{tabular}


(100\%) estavam positivas para pelo menos um sorovar, e a prevalência em cada criação variou de $25 \%$ a $100 \%$.

Na análise univariada (Quadro 2), a detecção de anticorpos anti-Leptospira spp. mostrou-se relacionada aos seguintes fatores de risco (significância estatística $\mathrm{p}<0,05$ ): sistema de criação em confinamento e semiconfinamento, alimentação que incluía lavagem, contato com suínos de outros criatórios, presença de roedores e ocorrência de abortos. Criações com menos de 100 animais e o uso de água tratada se mostraram como fatores de proteção.

Após ajustes das variáveis, no modelo final de regressão logística multivariada, os fatores de risco que tiveram associação com a ocorrência da infecção por Leptospira spp. foram o sistema de criação de confinamento e o saneamento ruim das instalações. 0 uso de água tratada permaneceu como fator de proteção (Quadro 3).

Quadro 2. Número de reações às sorovariedades de Leptospira spp. pelo teste de soroaglutinação microscópica, entre os 412 suínos abatidos no período de janeiro a dezembro de 2013, de acordo com as variáveis pesquisadas, e os respectivos valores de odds ratio, intervalo de confiança e significância estatística. Mossoró/RN, 2013

\begin{tabular}{|c|c|c|c|c|c|}
\hline Variável & + & - & $\mathrm{OR}$ & IC-95\% & $\mathrm{p}$ \\
\hline \multicolumn{6}{|l|}{ Faixa etária animais } \\
\hline Jovem & 184 & 42 & 1,439 & $0,897-2,309$ & 0,130 \\
\hline Adulto & 140 & 46 & 1 & & \\
\hline \multicolumn{6}{|c|}{ Zoonoses - conhecimento do proprietário } \\
\hline Sim & 48 & 7 & 2,012 & $0,877-4,619$ & 0,093 \\
\hline Não & 276 & 81 & 1 & & \\
\hline \multicolumn{6}{|c|}{ Suinocultura como atividade principal } \\
\hline Sim & 242 & 58 & 1,526 & $0,919-2,534$ & 0,101 \\
\hline Não & 82 & 30 & 1 & & \\
\hline \multicolumn{6}{|l|}{ Sistema de criação } \\
\hline Confinamento & 124 & 29 & 12,83 & $3,266-50,38$ & $0,000^{*}$ \\
\hline Semiconfinamento & 197 & 50 & 11,82 & $3,085-45,29$ & $0,000^{*}$ \\
\hline Extensivo & 03 & 9 & 1 & - & - \\
\hline \multicolumn{6}{|l|}{ Total suínos } \\
\hline$<100$ & 58 & 35 & 0,330 & $0,198-0,551$ & $0,000^{*}$ \\
\hline$\geq 100$ & 266 & 53 & 1 & & \\
\hline \multicolumn{6}{|l|}{ Instalações } \\
\hline Madeira e terra & 243 & 73 & 0,616 & $0,335-1,134$ & 0,118 \\
\hline Alvenaria, madeira e terra & 81 & 15 & 1 & & \\
\hline \multicolumn{6}{|l|}{ Saneamento } \\
\hline Ruim & 135 & 32 & 1,665 & $0,817-3,392$ & 0,179 \\
\hline Regular & 151 & 41 & 1,454 & $0,728-2,899$ & 0,286 \\
\hline Bom & 38 & 15 & 1 & - & - \\
\hline \multicolumn{6}{|l|}{ Alimentação com lavagem } \\
\hline Sim & 273 & 65 & 1,894 & $1,080-3,322$ & $0,024^{*}$ \\
\hline Não & 51 & 23 & 1 & & \\
\hline \multicolumn{6}{|l|}{ Água tratada } \\
\hline Sim & 76 & 40 & 0,368 & $0,225-0,601$ & $0,000^{*}$ \\
\hline Não & 248 & 48 & 1 & & \\
\hline \multicolumn{6}{|l|}{ Higiene diária } \\
\hline Sim & 90 & 18 & 1,496 & $0,844-2,651$ & 0,166 \\
\hline Não & 234 & 70 & 1 & & \\
\hline \multicolumn{6}{|l|}{ Contato outros suínos } \\
\hline Sim & 139 & 25 & 1,893 & $1,134-3,162$ & $0,014^{*}$ \\
\hline Não & 185 & 63 & 1 & & \\
\hline \multicolumn{6}{|l|}{ Caprino/Ovino } \\
\hline Sim & 164 & 35 & 1,552 & $0,961-2,506$ & 0,071 \\
\hline Não & 160 & 53 & 1 & & \\
\hline \multicolumn{6}{|l|}{ Ratos } \\
\hline Sim & 232 & 49 & 2,007 & $1,236-3,260$ & $0,004^{*}$ \\
\hline Não & 92 & 39 & 1 & & \\
\hline \multicolumn{6}{|l|}{ Aborto } \\
\hline Sim & 151 & 26 & 2,081 & $1,253-3,457$ & $0,004^{*}$ \\
\hline Não & 173 & 62 & 1 & & \\
\hline
\end{tabular}

* Significância estatística (p $<0,05)$, OR = Odds Ratio, IC-95 = intervalo de confiança de 95\%. 
Quadro 3. Variáveis que permaneceram no modelo final da análise multivariada para os casos de leptospirose em suínos, e os respectivos valores de odds ratio, intervalo de confiança e significância estatística. Mossoró/RN, 2013

\begin{tabular}{lclc}
\hline \multicolumn{1}{c}{ Variável } & Odds ratio & \multicolumn{1}{c}{ IC-95\% } & $\mathrm{p}$ \\
\hline Sistema de confinamento & 33,794 & $7,762-147,129$ & 0,000 \\
Saneamento ruim & 2,382 & $1,275-4,448$ & 0,006 \\
Água tratada & 0,169 & $0,085-0,337$ & 0,000
\end{tabular}

$\overline{\text { Hosmer e Lemeshow }}\left(\chi^{2}=2,110, \mathrm{gl}=07, \mathrm{p}=0,953\right)$; IC-95 = intervalo de confiança de $95 \%$.

\section{DISCUSSÃO}

0 presente estudo foi o primeiro levantamento soroepidemiológico de Leptospira spp. em suínos no município de Mossoró, Estado do Rio Grande do Norte. Os resultados encontrados demonstraram uma elevada prevalência $(78,6 \%)$, superior aos valores descritos na literatura brasileira, na região Sudeste descrita por Ramos \& Lilenbaum (2002) com 66,1\% de animais sororreagentes no Estado do Rio de Janeiro e por Shimabukuro et al. (2003) (36,6\%), Azevedo et al. (2006) $(16,5 \%)$ e Silva et al. (2010) (41\%) em São Paulo; na região Centro-oeste, no Estado de Goiás com 65,2\% (Souza 2000); no Sul do país no Estado do Rio Grande do Sul (37,9\%) (Favero et al. 2002) e Paraná (28,0\%) (Hashimoto et al. 2010); na região Norte em Rondônia (32,9\%) (Aguiar et al. 2006); e na região Nordeste no Estado de Pernambuco (25,6\%) (Cavalcanti 2011), Piauí e Maranhão (4,7\%) (Gonçalves et al. 2011), Alagoas (16,1\%) (Valença et al. 2013) e Paraíba (14,6\%) (Figueiredo et al. 2013).

As propriedades com pelo menos um animal positivo no teste de SAM, ou seja, com títulos maiores ou iguais a 100, foram consideradas positivas. Os resultados demonstraram que a leptospirose estava disseminada em todas as propriedades examinadas do município e com elevado percentual, comprovando assim a endemicidade da infecção em suínos do município de Mossoró no Estado do Rio Grande do Norte. Achados semelhantes foram encontrados por Valença et al. (2013) em todas as granjas de suínos estudadas no Estado de Alagoas, no entanto, a frequência de animais positivos por fazenda foi menor, variando de $4,0 \%$ a $24,3 \%$.

Os resultados também demonstraram que no município foram constatados animais reatores a 15 sorovares de Leptospira spp., sugerindo uma grande variação de reservatórios nos locais de origem dos animais. Nos estudos realizados no Brasil, o número de sorovares foi menor que o encontrado na presente pesquisa. Até então, Souza (2000) verificou a ocorrência de reações a 11 sorovares em granjas de suínos no Estado de Goiás. Houve elevada ocorrência de coaglutinações (22,3\%) entre as amostras examinadas. Estas podem ser explicadas, como apontado por Marques et al. (2010), pela infecção concomitante de vários sorovares de Leptospira spp. ou por reações cruzadas entre sorovares de um mesmo sorogrupo.

De acordo com Soto et al. (2007), os principais sorovares diagnosticados em suínos no Brasil pela SAM foram: Pomona, Icterohaemorrhagiae, Grippotyphosa, Pyrogenes, Canicola, Autumnalis e Javanica. Porém no presente estudo não foram identificados os sorovares Grippotyphosa, Pyrogenes e Javanica. O sorovar Icterohaemorrhagiae foi o mais prevalente entre os suínos investigados. 0 resultado revela similaridade com outras pesquisas, conforme encontrado por Ramos e Lilenbaum (2002) em criações tecnificadas no Estado do Rio de Janeiro; Favero et al. (2002) em Minas Gerais, Goiás, Paraná, Santa Catarina e São Paulo; Delbem et al. (2004) no Paraná; Osava et al. (2010) em Minas e Goiás; Gonçalves et al. (2011) no Piauí e Maranhão; e Valença et al. (2013) em Alagoas.

0 sorovar Icterohaemorrhagiae, bem como Castellonis, Copenhageni e Autumnalis, em que houve suínos reagentes, tem nos roedores os seus hospedeiros mais comuns (Faine et al. 1999, Aguiar et al. 2006, Valença et al. 2013), os quais podem ser indicados como responsáveis pela presença deste agente nas instalações das criações. Convém salientar que os suínos apresentavam características de hospedeiro de manutenção do sorovar Icterohaemorrhagiae, pois não havia sinais clínicos ou qualquer outra manifestação da doença. 0 sorovar Pomona foi o segundo mais frequente entre suínos reatores, acometendo 46 animais. Os suínos são hospedeiros de manutenção deste sorovar, assim como dos sorovares Bratislava e Tarassovi, também encontrados na presente pesquisa.

O sorovar Shermani foi o terceiro mais frequente, entre os suínos reatores, diagnosticado em 32 animais. Nos estudos de soroprevalência realizados no Brasil, esta sorovariedade foi pouco encontrada em suínos; apenas Azevedo et al. (2006) o identificaram em uma granja no Estado de São Paulo. Os maiores disseminadores do sorovar Shermani são alguns mamíferos, entre eles os roedores silvestres infectados (Sulzer et al. 1982). Sua presença causa preocupação, pois não existe imunidade cruzada entre as diferentes sorovariedades, e as vacinas polivalentes para a prevenção da leptospirose disponíveis no mercado são compostas basicamente pelos sorovares Icterohaemorrhagiae, Canicola, Hardjo, Grippothyphosa, Pomona, Bratislava e Tarassovi (Figueiredo et al. 2013). 0 achado reforça ainda mais a importância da pesquisa continuada no desenvolvimento de novas vacinas antileptospirose mais efetivas e de imunidade mais duradoura, bem como a necessidade da inclusão de novas sorovariedades. Convém destacar também que, além do sorovar Shermani, boa parte das 15 variedades sorológicas em que houve animais reatores não são contempladas nas vacinas disponíveis no mercado brasileiro.

O sorovar Patoc, diagnosticado em 14 (3,4\%) animais sororreagentes, foi o quarto mais frequente. É um sorovar de Leptospira biflexa, apatogênico e de vida livre, utilizado como marcador sorológico, pois costuma reagir precocemente, e apresenta reações cruzadas com sorovares patogênicos (Aguiar et al. 2010). Outros sorovares aos quais houve animais reatores, porém em menor frequência, como Sentot, Bataviae, Butembo, Australis, Panama e Canicola, têm a sua importância por fornecerem informações sobre as variantes sorológicas que atuam na região. 0 sorovar Canicola é mantido por cães (Mello \& Manhoso 2007), e estes animais estavam presentes em boa parte das propriedades pesquisadas e tinham livre acesso às diversas criações animais.

Estudos sobre os principais fatores de risco associados com a leptospirose suína no Brasil são escassos. Alguns trabalhos destacaram a não realização de quarentena, a inseminação artificial, a ocorrência de roedores nas granjas, o manejo da água dentro das criações, a presença de áreas alagadiças, o número de matrizes no plantel, o nível de tecnificação, as falhas na higienização do reservatório de água e o controle de roedores (Boqvist et al. 2002, Delbem et al. 2004, Valença et al. 2013). 
No presente estudo, o sistema de confinamento apresentou-se como um importante fator de risco para a ocorrência da infecção por Leptospira spp., sendo a chance de um animal em confinamento se infectar 33,8 vezes maior quando comparado aos criados de forma extensiva. A densidade da população de animais, em geral alta nos sistemas de confinamento, assume característica epidemiológica fundamental que influi na presença da leptospirose suína. À medida que aumenta o número de indivíduos por unidade de superfície, aumenta o risco de exposição por contato com a fonte comum. Um pequeno número de portadores em um ambiente úmido pode contaminar rapidamente todo o meio, tornando-se difícil que um indivíduo escape da exposição (Szyfres 1976). Ao contrário, Campos et al. (2011) e Gonçalves et al. (2011), comparando os dois sistemas de criação quanto à predisposição para contrair infecção por Leptospira spp., revelaram que a suscetibilidade foi maior nos animais criados extensivamente do que nos de criação confinada.

Os fatores de risco identificados para a ocorrência desta enfermidade compreendem aspectos ligados às falhas no saneamento e à promiscuidade entre as espécies animais, mostrando serem facilitadores na disseminação dos agentes infecciosos e devendo ser ajustados para o controle da infecção nos rebanhos estudados. 0 exposto é justificado, uma vez que o acúmulo de urina, água, restos de alimentos e fômites são importantes na cadeia epidemiológica de transmissão da infecção. A prevenção da leptospirose suína é largamente dependente de medidas de saneamento dos criatórios que, muitas vezes, são difíceis de serem implementadas, principalmente em regiões onde a suinocultura não é tecnificada (Delbem et al. 2004). Gonçalves \& Costa (2011) citaram como medidas de saneamento das criações suínas os programas de desinfecção que incluam a realização de vazio sanitário (tudo dentro, tudo fora) para a eliminação de leptospiras que possam estar presentes nas instalações.

A água tratada demonstrou ser fator de proteção para a leptospirose. 0 achado é coerente, pois do contrário a água estaria mais propensa à contaminação, podendo funcionar como uma das principais vias de transmissão de patógenos aos animais domésticos, constituindo um fator importante, tanto para a economia, por poder acarretar prejuízos econômicos, como para a saúde pública, pois muitos dos seus agentes causais podem ser transmitidos ao ser humano (Amaral et al. 2005). Delbem et al. (2004), estudando os fatores de risco de leptospirose suína, constataram que os aspectos relacionados aos reservatórios e a drenagem da água nas criações estavam associados à ocorrência da infecção.

Alguns problemas eram comuns a todas as propriedades estudadas, independentemente de os suínos serem positivos ou negativos para leptospirose. A presença de moscas, a não segregação dos animais por faixa etária, o manejo reprodutivo sem controle (monta natural), a ausência de área destinada a animais em quarentena ou doentes, a não vacinação contra a leptospirose, a ausência de assistência veterinária regular e a carência de informações por parte dos proprietários foram achados regulares, mas não incluídos como objeto de análise.

No presente trabalho, os animais reatores não apresentavam sinais clínicos da doença, o que é importante do ponto de vista epidemiológico, pois os animais assintomáticos podem eliminar constantemente o agente, garantindo a sua persistência no ambiente (Vasconcellos 1993, Faine et al. 1999). Assim, podem atuar como potentes fontes de infecção, já que nesta espécie a leptospirúria pode durar até meses, expondo os trabalhadores das criações ao perigo da doença ocupacional. Os resultados são importantes, uma vez que os prejuízos econômicos causados pelos agentes etiológicos da leptospirose são decisivos para a sustentabilidade das criações, além de poder ocasionar riscos significativos em termos de saúde pública.

Conhecer a suinocultura local nos seus aspectos sanitários e o potencial de risco de zoonoses nos rebanhos reveste-se de grande relevância no cenário atual. 0 conhecimento dos aspectos sanitários, de seus indicadores e de potenciais determinantes do processo saúde-doença neste contexto pode orientar o planejamento e a avaliação das ações de sanidade animal. Além de assegurar o uso racional dos recursos e, sobretudo, favorecer o alcance de melhores condições do rebanho e, consequentemente, ganhos econômicos para o produtor. Para tanto, torna-se necessária a difusão de conhecimento para os criadores de suínos, com base na realidade local, que envolva os órgãos de defesa sanitária animal, de meio ambiente e saúde pública. E ainda por meio de ações de capacitação técnica, educação sanitária, abastecimento de insumos, melhorias das instalações e organização da produção, obter melhorias na produção, tornando a atividade menos vulnerável do ponto de vista sanitário e consequentemente econômico.

\section{CONCLUSÕES}

Pode-se afirmar que a infecção por Leptospira spp. está disseminada em explorações suinícolas no município de Mossoró, Rio Grande do Norte, com elevada prevalência nos animais e nas propriedades.

Foi encontrado grande número de animais sororreatores a um grande número de sorovares, com indicativo de roedores como responsáveis pela presença do principal agente (sorovar Icterohaemorrhagiae) nas instalações das criações, caracterizando promiscuidade entre as espécies hospedeiras.

Os fatores de risco identificados neste estudo compreenderam aspectos ligados à aglomeração de animais e falhas no saneamento, mostrando serem facilitadores na disseminação do agente infeccioso, devendo ser ajustados para controlar a infecção nos rebanhos estudados.

As respostas sorológicas aos sorovares testados apontaram para a necessidade de composições mais apropriadas de vacinas para o rebanho local, uma vez que a maioria das vacinas comerciais não incluem os antígenos para Shermani e Autumnalis, que foram prevalentes entre os animais deste estudo.

Os achados levantam preocupações do ponto de vista de saúde pública, uma vez que os profissionais envolvidos no manejo e no abate desses animais estão expostos ao risco de infecção.

\section{REFERÊNCIAS}

Acha P.N. \& Szyfres B. 2001. Zoonosis y Enfermedades Transmisibles Comunes al Hombre y a los Animales. 3ํㅡㄹ ed. Publicación Científica 398, OPS, Washington, DC. p. 175-186

Aguiar D.M., Cavalcante G.T., Dib C.C., Villalobos E.M.C., Cunha E.M.S., Lara M.C.C.S.H., Rodriguez C.A.R., Vasconcellos S.A., Moraes Z.M., Labruna M.B., Camargo L.M.A. \& Gennari S.M. 2006. Anticorpos contra agentes bacterianos e virais em suínos de agricultura familiar do município de Monte Negro, RO. Arqs Inst. Biológico, São Paulo, 73(4):415-419.

Aguiar D.M., Cavalcante G.T., Vasconcellos S.A., Souza G.O., Labruna M.B., Camargo L.M.A. \& Gennari S.M. 2010. Anticorpos anti-Leptospira spp. em ovinos do município de Monte Negro, estado de Rondônia. Arqs Inst. Biológico, São Paulo, 77(3):529-532. 
Amaral L.A., Rossi Junior O.D., Nader Filho A., Souza M.C.I. \& Isa H. 2005. Água utilizada em suinocultura como fator de risco à saúde humana e animal. Ars Vet. 21:41-46.

Araújo Neto J.O., Alves C.J., Azevedo S.S., Silva M.L.C.R. \& Batista C.S.A. 2010. Soroprevalência da leptospirose em caprinos da microrregião do Seridó Oriental, Estado do Rio Grande do Norte, Brasil, e pesquisa de fatores de risco. Braz. J. Vet. Res. Anim. Sci. 47(2):144-155. http://dx.doi.org/10.11606/ issn.1678-4456.bjvras.2010.26839.

Azevedo S.S., Alves C.J., Andrade J.S.L., Batista C.S.A., Clementino I.J. \& Santos F.A. 2004. Ocorrência de aglutininas anti-Leptospira em ovinos do estado do Rio Grande do Norte, Brasil. Revta Bras. Ciênc. Vet. 11(3):167-170.

Azevedo S.S., Soto F.R.M., Morais Z.M., Pinheiro S.R., Vuaden E.R., Batista C.S.A., Souza G.O., Delbem A.C.B., Gonçales A.P. \& Vasconcellos S.A. 2006. Frequency of anti leptospires agglutinins in sows from a swine herd in the Ibiúna Municipality, State of São Paulo, Brazil. Arqs Inst. Biológico, São Paulo, 73(1):97-100.

Boqvist S., Thu H.T.V., Vagsholm I. \& Magnusson U. 2002. The impact of Leptospira seropositivity on reproductive performance in sows in southern Viet Nam. Theriogenology 58(7):1327-1335. http://dx.doi.org/10.1016/ S0093-691X(02)00971-8. PMid:12387346.

Brasil 2009. Guia de Vigilância Epidemiológica. 7ª̣ ed. Brasília, Ministério da Saúde. p. 15-32.

Campos A.P., Gonçalves L.M.F., Freire S.M., Leal L.M., Mineiro A.L.B.B. \& Costa F.A.L. 2011. Aglutininas antileptospiras em suínos abatidos para consumo e associação ao comprometimento hepático e pulmonar. Revta Patol. Trop. 40(2):137-148. http://dx.doi.org/10.5216/rpt.v40i2.14939.

Cavalcanti E.F.T.S.F. 2011. Pesquisa de Toxoplasma gondii e anticorpos antiLeptospira spp. em suínos abatidos no Agreste do Estado de Pernambuco, Brasil. Dissertação de Mestrado em Ciência Veterinária, Universidade Federal Rural de Pernambuco, Recife, PE. 82p.

Delbem A.C.B., Freire R.L., Silva C.A., Müller E.E., Dias R.A., Ferreira Neto J.S. \& Freitas J.C. 2004. Fatores de risco associados à soropositividade para leptospirose em matrizes suínas. Ciência Rural 34(3):847-852. http:// dx.doi.org/10.1590/S0103-84782004000300029.

Faine S., Adler B., Bolin C. \& Perolat P. 1999. Leptospira and Leptospirosis. 2nd ed. MediSci., Melbourne, p.34-38.

Favero A.C.M., Pinheiro S.R., Vasconcellos S.A., Morais Z.M., Ferreira F. \& Ferreira Neto J.S. 2002. Sorovares de leptospiras predominantes em exames sorológicos de bubalinos, ovinos, caprinos, eqüinos, suínos e cães de diversos estados brasileiros. Ciência Rural 32(4):613-619. http://dx.doi. org/10.1590/S0103-84782002000400011.

Favero M., Pinheiro S.R., Vasconcellos S.A., Morais Z.M., Ferreira F. \& Ferreira Neto J.S. 2001. Leptospirose bovina, variantes sorológicas predominantes em colheitas efetuadas no período de 1984 a 1997 em rebanhos de 21 estados do Brasil. Arqs Inst. Biológico, São Paulo, 68(2):29-35.

Figueiredo Í.L., Higino S.S.S., Alves C.J., Del Fava C., Carretero M.E. \& Azevedo S.S. 2013. Interrelação entre frequência de anticorpos anti-leptospira spp. e exames histopatológicos (hematoxilina-eosina e warthin-starry) em suínos abatidos no Semiárido Paraibano. Arqs Inst. Biológico, São Paulo, 80(1):27-34. http://dx.doi.org/10.1590/S1808-16572013000100005.

Gonçalves L.M.F. \& Costa F.A.L. 2011. Leptospiroses em suínos no Brasil. Revta Patol. Trop. 40(1):1-14.

Gonçalves L.M.F., Mineiro A.L.B.B., Carvalho S.M., Campos A.P., Evangelista L.S.M., Pinho F.A., Moreira E.C. \& Costa F.A.L. 2011. Pesquisa de aglutininas, antígeno de leptospiras e apoptose em rim de suínos naturalmente infectados por Leptospira spp. Pesq. Vet. Bras. 31(7):561-568. http:// dx.doi.org/10.1590/S0100-736X2011000700003.

Hashimoto V.Y., Garcia J.L., Spohr K.A.H., Silva F.G., Alves L.A. \& Freitas J.C. 2010. Prevalência de anticorpos contra Leptospira spp. em bovinos, caninos, equinos, ovinos e suínos do município de Jaguapitã, estado do Paraná, Brasil. Arqs Inst. Biológico, São Paulo, 77(3):521-524.

Higgins R. 2004. Emerging or re-emerging bacterial zoonotic diseases: bartonellosis, leptospirosis, lyme borreliosis, plague. Rev. Scient. Tech.
Off. Int. Epizoot., Paris, 23(2):569-581. http://dx.doi.org/10.20506/ rst.23.2.1503. PMid:15702720.

IBGE 2012a. Produção da Pecuária Municipal. Vol. 40. Instituto Brasileiro de Geografia e Estatística, Rio de Janeiro, p.1-71.

IBGE 2012b. Censo demográfico 2010: resultados gerais da Amostra. Instituto Brasileiro de Geografia e Estatística, Rio de Janeiro.

Marques A.E., Rocha W.V., De Brito W.M.E.D., Fioravanti M.C.S., Parreira I.M. \& Jayme V.D.S. 2010. Prevalência de anticorpos anti-Leptospira spp. e aspectos epidemiológicos da infecção em bovinos do Estado de Goiás. Ciênc. Anim. Bras. 11(3):607-617. http://dx.doi.org/10.5216/cab.v11i3.5460.

Mello L.P.P. \& Manhoso F.F.R. 2007. Aspectos epidemiológicos da leptospirose canina no Brasil. Unimar Ciências 16:27-32.

OIE 2008. Manual of Diagnostic Test and Vaccines for Terrestrial Animals World Organization for Animal Health. Disponível em <http://www.oie. int/fileadmin/Home/eng/Health_standards/tahm/2.01.09_LEPTO.pdf> Acesso em 6 nov. 2014.

Osava C.F., Salaberry S.R.S., Nascimento C.C.N., Lima-Ribeiro A.M.C., Moreira R.Q., Castro J.R. \& Rigo V.H.B. 2010. Ocorrência de anticorpos anti-Leptospira spp. em diferentes sistemas de criação de suínos. Biosci. J. 26(2):202-207.

Ramos A.C.F. \& Lilenbaum W. 2002. Fatores que influenciam a ocorrência de aglutininas anti-Leptospira em suínos de criação tecnificada do estado do Rio de Janeiro. Revta Bras. Med. Vet. 24(2):20-29.

Shimabukuro F.H., Domingues P.F. \& Langoni H. 2003. Searching of swine leptospiral carrier by microbial isolation and polymerase chain reaction in kidney samples from serologically positive and negative animals. Braz. J. Vet. Res. Anim. Sci. 40(4):243-253.

Silva F.J., Mathias L.A., Magajevski F.S., Werther K., Assis N.A. \& Girio R.J.S. 2010. Anticorpos contra Leptospira spp. em animais domésticos e silvestres presentes no campus universitário da FCAV, Unesp, Jaboticabal/SP. Ars Vet. 26(1):17-25.

Soto F.R.M., Vasconcellos S.A., Pinheiro S.R., Bernarsi F. \& Camargo S.R. 2007. Artigo de revisão: leptospirose suína. Arqs Inst. Biológico, São Paulo, 74:379-395.

Souza A.S. 2000. Estudo da prevalência de Leptospira interrogans em reprodutores suínos em produção e aspectos epidemiológicos da infecção em Goiás. Dissertação de Mestrado em Ciência Animal, Escola de Veterinária, Universidade Federal de Goiás, Goiânia. 74p.

Sulzer K., Pope V. \& Rogers F. 1982. New leptospiral serotypes (serovars) from the Western Hemisphere isolated during 1964 through 1970. Revta Latinoam. Microbiol. 24(1):15-17. PMid:7186673.

Szyfres B. 1976. La leptospirosis como problema de salud humana y animal em America Latina y el area del Caribe. Annales VII Reunião Interamericana Sobre el Control de la Fiebre Aftosa y Otras Zoonosis, Publicación Científica 316, Organización Panamericana de la Salud, Guatemala. 189p.

Thrusfield M.V. 2004. Epidemiologia Veterinária. $2^{a}$ ed. Roca, São Paulo. p. 223-247.

Valença R.M.B., Mota R.A., Castro V., Anderlini G.A., Pinheiro Júnior J.W., Brandespim D.F., Valença S.R.F.A. \& Guerra M.M.P. 2013. Prevalence and risk factors associated with Leptospira spp. infection in technified swine farms in the State of Alagoas, Brazil. Transbound. Emerg. Dis. 60(1):79-86. http://dx.doi.org/10.1111/j.1865-1682.2012.01320.x. PMid:22469011.

Vasconcellos S.A. 1993. Leptospirose animal. Anais III Encontro Nacional em Leptospirose, Rio de Janeiro, RJ, p.62-66.

Vieira A.S., Rosinha G.M.S., Vasconcellos S.A., Moaris Z.M., Viana R.C., Oliveira C.E., Soares C.O., Araújo F.R., Mourão G.M., Bianchi R.C., Olifiers N., Rademaker V., Rocha F.L. \& Pellegrin A.O. 2013. Identificação de mamíferos silvestres do Pantanal Sul-Mato-Grossense portadores de Leptospira spp. Ciênc. Anim. Bras. 14(3):373-380. 